\title{
Functional Characterization of TvCyt2, a Member of the p450 Monooxygenases From Trichoderma virens Relevant During the Association With Plants and Mycoparasitism
}

\author{
Claudia A. Ramírez-Valdespino,, ${ }^{1}$ Maria Daniela Porras-Troncoso, ${ }^{1}$ Alma Rosa Corrales-Escobosa, ${ }^{2}$ \\ Kazimierz Wrobel, ${ }^{2}$ Pedro Martínez-Hernández, ${ }^{3}$ and Vianey Olmedo-Monfil ${ }^{1, \dagger}$ \\ ${ }^{1}$ Universidad de Guanajuato, División de Ciencias Naturales y Exactas, Departamento de Biología, Guanajuato, Gto. México; \\ ${ }^{2}$ Universidad de Guanajuato, División de Ciencias Naturales y Exactas, Departamento de Química, Guanajuato, Gto. México; \\ and ${ }^{3}$ Laboratorio Nacional de Genómica para la Biodiversidad, Centro de Investigación y Estudios Avanzados del IPN, Irapuato, \\ Gto. México
}

Accepted 5 October 2017.

\begin{abstract}
Secondary metabolites are crucial for the establishment of interactions between plants and microbes, as in the case of Trichoderma-plant interactions. In the biosynthetic pathway of secondary metabolites, specific enzymes participate in the formation of hydroxyl and epoxy groups, belonging to the p450 monooxygenases family. Here, we show that the product of the gene TvCyt2 from Trichoderma virens encodes a new protein homologous to the cytochrome p450, which is downregulated at the beginning of Trichoderma-Arabidopsis interaction. To investigate its role in the interactions established by Trichoderma spp., we analyzed the metabolic profile obtained from the overexpressing (OETvCyt2) and null mutant ( $\Delta t v c y t 2)$ strains, observing that the OETvCyt2 strains produce a higher concentration of some metabolites than the wild-type (WT) strain. $\Delta t v c y t 2$ strains showed a decreased antagonistic activity against Rhizoctonia solani in antibiosis assays. Arabidopsis plants cocultivated with the OETvCyt2 strains showed stronger induction of systemic acquired resistance than plants cocultivated with the WT strain, as well as increases in biomass and fitness. Our data suggest that the product of the TvCyt2 gene is involved in secondary metabolite biosynthesis, which can increase antagonistic activity with phytopathogenic fungi and the capacity to promote plant growth.
\end{abstract}

Plants and microorganisms establish molecular dialogs that involve recognition of conserved signaling molecules that play key roles during this communication (Zeilinger et al. 2016). Some of these molecules are produced by secondary metabolism, determining the type of association to be established, either beneficial or harmful interactions (Bais et al. 2006; Ortíz-Castro et al. 2009). The pathways leading to the synthesis of secondary metabolites include the participation of different enzymatic activities.

Among the main enzymatic activities involved in the biosynthesis of secondary metabolites are the polyketide synthases, nonribosomal peptide synthases, dimethylallyltryptophan synthases, and cytochrome p450 monooxigenases (CYP) (Werck-Reichhart

${ }^{\dagger}$ Corresponding author: Vianey Olmedo-Monfil;

E-mail:vg.olmedo@ugto.mx

*The $\boldsymbol{e}$-Xtra logo stands for "electronic extra" and indicates that nine supplementary figures are published online.

@ 2018 The American Phytopathological Society and Feyereisen 2000; Mukherjee et al. 2006; Deepika et al. 2016). In the fungal kingdom, CYPs have attracted attention due to reports linking them with new industrial and biotechnological applications. In fungi, CYPs participate in primary and secondary metabolism and are involved in detoxification and degradation of toxic compounds (Durairaj et al. 2016). This class of enzymes catalyze regio- and stereospecific conversions of a wide range of lipophilic compounds to more hydrophilic derivatives by introducing an oxygen atom and, in some cases, p450 enzymes can catalyze hydroxylation or epoxidation reactions (Črešnar and Petrič 2011).

The production of secondary metabolites, regarding quantity and time, is crucial for triggering a perception and interaction in biological associations (Sharma et al. 2017; Vinale et al. 2016; Zhai et al. 2017). According to the role of these molecules during plant-fungus interactions, some are identified as effector-like molecules because they are capable of altering the host cell structure and function either facilitating infection or triggering defense responses (Hogenhout et al. 2009). In Magnaporthe oryzae, a polyketide synthase encoded by the $A C E 1$ gene is related to the synthesis of secondary metabolites that might function as effectors recognized by resistance proteins (Collemare et al. 2008; Yi and Valent 2013). In Colletotrichum higginsianum, a gene cluster implicated in secondary metabolite biosynthesis is reported; compounds produced by this pathway are predicted to function in host-response manipulation, as protein effectors do (O'Connell et al. 2012). In nonpathogenic fungus, little is known about the role of effector molecules and, therefore, secondary metabolite involvement in plant-fungi interactions.

Trichoderma species are known, because of their use as biological control agents, to antagonize fungal plant pathogens. This characteristic is mainly attributed to the production of metabolites with antimicrobial activity and the secretion of several hydrolytic enzymes (Lorito et al. 2010). Furthermore, Trichoderma spp. establish interactions with plants, colonizing the epidermis and root cortex, this penetration being restricted to the apoplast as a consequence of the cell-wall reinforcement (Chacón et al. 2007). The interaction enhances root and plant growth as well as activates the defense responses mediated by salicylate (SA), jasmonate (JA), and ethylene (ET) (Contreras-Cornejo et al. 2009; Salas-Marina et al. 2011). Trichoderma spp. are able to establish such interactions, inducing massive changes at transcriptomic and metabolomic level (Morán-Diez et al. 2015; Rubio et al. 2012), and some of these metabolites have been found, not only to directly inhibit the growth of pathogenic microorganisms, but also increase 
disease resistance triggering the defense system in plants (induced systemic resistance) (Vos et al. 2015). Furthermore, metabolitepretreated plants responded to a pathogen attack much faster or more intensively (Hermosa et al. 2012; Shoresh et al. 2010; Verhagen et al. 2011), a mechanism known as priming (Conrath 2011).

During the establishment of the association of Trichoderma spp. with plants, a specific molecular communication, as in other biological interactions, must be involved, and these molecules either are recognized or trigger, or both, physiological changes in both organisms, allowing the establishment of the association. Among the compounds released by Trichoderma spp., which are related to the interaction with plants, effector-like molecules have been reported, such as cellulases, cerato-platanins, and secondary metabolites such as antibiotics, hormones, mycotoxins, and terpenes (Atanasova et al. 2013; Brito et al. 2014; Hermosa et al. 2012; Horwitz et al. 2013). In the interaction of Trichoderma spp. with common bean (Phaseolus vulgaris), tomato (Solanum lycopersicum), and Arabidopsis thaliana, the presence of the fungus promotes accumulation of pathogenesis-related proteins (Salas-Marina et al. 2011; Woo et al. 2006). Contreras-Cornejo and collaborators (2011) observed that the presence of $T$. virens increases the levels of SA and JA in A. thaliana.

In other Trichoderma species, the participation of secondary metabolites during plant-Tichoderma interactions has been demonstrated. In T. brevicompactum, the overexpression of the gene tri5, which encodes for a p450 protein, increased the production levels of trichodermin and other metabolites, leading to an exacerbated antimicrobial activity (Tijerino et al. 2011).

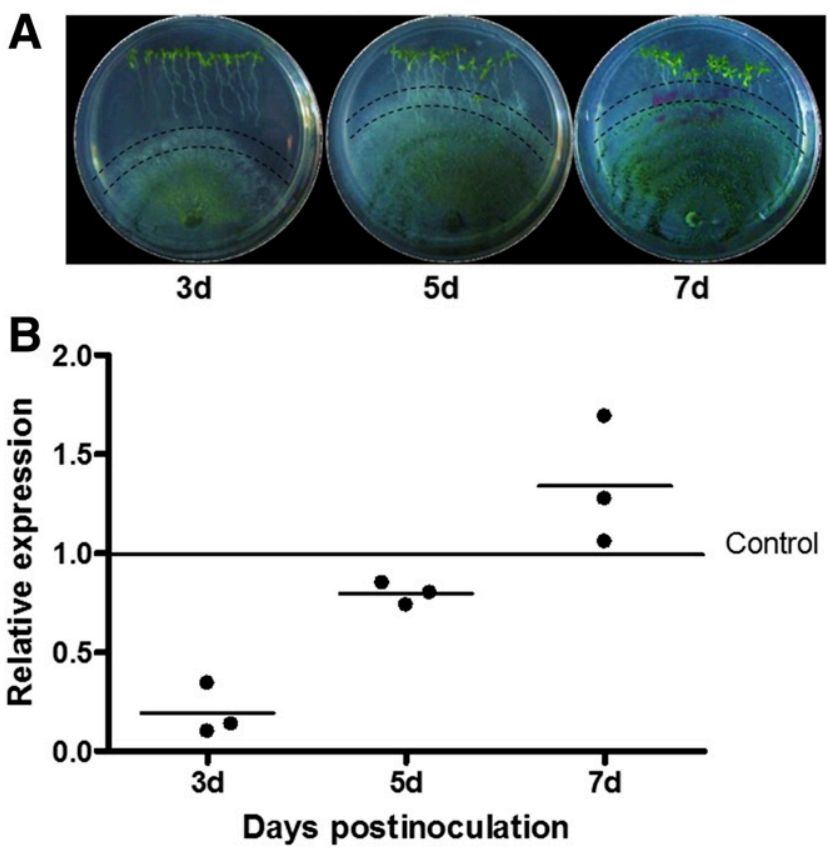

Fig. 1. The expression of $T v C y t 2$ from Trichoderma virens is down-regulated in the earlier interaction time with Arabidopsis thaliana. A, Representative photograph of Arabidopsis seedlings cocultivated with $T$. virens at 3, 5 and 7 days. Mycelia between the dashed lines were collected to the expression assays and Trichoderma cultured alone was used as control, collecting a similar quantity of mycelia. B, Determination of TvCyt2 expression by quantitative reverse transcription-polymerase chain reaction (qRT-PCR) in mycelia interacting with Arabidopsis at 3, 5 and 7 days. Glyceraldehyde-3-phosphate dehydrogenase gene ( $g p d)$ was used as endogenous expression control; for each time tested, the expression level determined for $T$. virens growing alone was considered as 1 . The qRT-PCR assay was done in triplicate with three technical replicates each. Data were analyzed using an analysis of variance nonparametric test with $P<0.05$. Only the expression of $T v C y t 2$ at 3 days had significant differences with respect to the control.
Studies on T. arundinaceum showed that biocontrol activity against Botrytis cinerea is, in part, due to harzianum A (HA), an analog of compounds belonging to a family of sesquiterpenes known as trichothecenes. HA is involved in the biocontrol activity by Trichoderma spp. as well as in its ability to upregulate plant defense-related genes (Malmierca et al. 2012). tri genes are also present in Fusarium species and are involved in the biosynthesis of mycotoxins (McCormick et al. 2004; Meek et al. 2003). The importance of the concentration of these molecules in the establishment of the interaction between Trichoderma spp. and plants is evidenced by the effect of harzianic acid and harzianolide, which either promote plant growth, induce plant resistance at low concentrations or both, while at high concentrations, they exhibit the opposite effect, inhibiting plant growth (Cai et al. 2013; Vinale et al. 2009).

In this work, we selected the $T$. virens gene $T v C y t 2$ from a catalog of effectors proposed by Guzmán-Guzmán and collaborators (2017), because $T \nu C y t 2$ is predicted to be a p450 family member that could be relevant in the establishment of biological interactions (Carpenter et al. 2008; Malmierca et al. 2012). We determined its differential transcriptional response in the presence of the plant host and fungal competitors. Additionally, we confirmed the participation of $T v C y t 2$ in the synthesis of secondary metabolites, by comparing metabolic profiles between wild-type (WT), mutants, and overexpressing strains of the $T v C y t 2$ gene. Moreover, we evaluated the impact of the differential production of secondary metabolites in antibiosis assays against phytopathogens. Finally, we determined their relevance during the establishment of symbiotic interactions with plants, focusing on plant defense responses and plant total mass.

\section{RESULTS}

In silico analysis and expression patterns of $T v C y t 2$ from $T$. virens.

To demonstrate if $T v C y t 2$ could participate during the biological associations established by $T$. virens with plants, we first analyzed its expression pattern in interactions with $A$. thaliana. Quantitative reverse transcription-polymerase chain reaction (qRT-PCR) assays using mycelia collected from the vicinity with A. thaliana seedlings, after 3, 5, and 7 days of interaction (Fig. 1A), showed that the gene was down-regulated at the first time of interaction, when there was not a physical contact between roots and hyphae; the $T v C y t 2$ expression level increased gradually during the two subsequent timepoints analyzed, until reaching the level shown by the control condition, in which $T$. virens was grown without plant interaction (Fig. 1B).

Trichoderma spp. are widely used as biocontrol agents because of their mycoparasitic activity against phytopathogenic fungi. To analyze if $T v C y t 2$ could be involved in this process, we determined expression levels of the $T v C y t 2$ gene in a confrontation with the anastomosis groups AG2 and AG5 of Rhizoctonia solani. Trichoderma mycelium was collected before contact between both fungi, during initial contact, and by the time Trichoderma overgrew the phytopathogens. $T v C y t 2$ expression was not modified in a confrontation with the AG2 strain. Interestingly, in confrontation with $R$. solani AG5, TvCyt 2 was up-regulated during the initial contact and overgrowth conditions (Fig. 2). These results suggest that the expression of $T v C y t 2$ gene depends on the phytopathogen with which $T$. virens is confronted.

The Joint Genome Institute database reports that the $T v C y t 2$ gene encodes for a protein member of the p450 family (ID 190045); the open reading frame is composed of $1,680 \mathrm{bp}$ and contains two introns that, after being processed, should generate a 1,581-bp sequence. Nevertheless, when the $T v C y t 2$ cDNA was amplified, cloned, and sequenced, we observed that the putative first intron is not processed, obtaining a sequence of 
$1,614 \mathrm{bp}$ that encodes for a protein of 537 amino acids, maintaining the open reading frame. The retained intron added $33 \mathrm{bp}$ and interrupted the region that encodes the putative signal peptide, indicating that it is not functional. Upon expression of the reporter $m$ Cherry fused to $T v C y t 2$ encoding sequence, red fluorescence was only observed inside the hypha (Supplementary Fig. S1). This result, together with the absence of the signal peptide and the predicted function, indicate that TvCyt2 protein is not secreted. The TvCyt 2 protein sequence contains five potential $\mathrm{N}$-glycosylation sites (analyzed with HIV database) and five predicted transmembrane domains (TMpred server and "DAS", the transmembrane prediction server), suggesting that the protein resides in some membrane, as is generally reported for $\mathrm{p} 450$ proteins, which are found primarily in the endoplasmic reticulum or the mitochondrial membrane (Park et al. 2014, 2015). The final deduced protein sequence has two characteristics found in p450 enzymes, an amino terminal domain (PERFL) related to membrane anchoring (Nebert and González 1987) and a motif (FXXGXXXCXC), which contains a conserved cysteine residue, as part of the heme-binding domain (Bairoch 1991; Larkin 1994) (Supplementary Fig. S2). We aligned the TvCyt 2 protein and observed that it has a similarity close to $75 \%$ with other hypothetical p450 proteins from Trichoderma species and less than $50 \%$ with some p450 from Trichoderma and Fusarium species (analyzed with Clustal Omega online) (Supplementary Fig. S3).

To analyze the presence of putative regulatory motifs in the promoter of $T v C y t 2$, a sequence 1,500 bp upstream of the ATG was analyzed. We found two CreA motifs for glucose repression, two ACEI recognition sequences for cellulose repression, and six mycoparasitic-related motifs MYC (Aro et al. 2001, 2003; Cortés et al. 1998; Cubero and Scazzocchio 1994; Lorito et al. 1996). We analyzed $T v C y t 2$ expression in culture media supplemented with glucose, cellulose, root exudates, or chitin. We did not observe a relevant change in the expression levels of $T v C y t 2$ (data not shown), suggesting that the expression of the gene could be affected for other molecules released by the fungus or the plant.

\section{TrCyt2 is involved in the biosynthesis of soluble secondary metabolites.}

To evaluate the possible role of TvCyt 2 in $T$. virens strains, overexpressing (OETvCyt2) and null mutants ( $\Delta t v c y t 2)$ were generated. For null mutants $T v C y t 2$ was replaced with the selection marker $h p h$, which confers hygromycin resistance. For overexpression, we generated a construct bearing $T v C y t 2$ gene under the control of the pyruvate kinase $(p k i)$ constitutive promoter from T. reesei (Yu et al. 2004; Zeilinger et al. 1999); gene replacement for the null mutants and construction integration for overexpression strains were confirmed by PCR (Supplementary Figs. S4 and S5). We selected two independent transformants for each type of strain and their $T v C y t 2$ expression levels were corroborated by qRT-PCR (Supplementary Fig. S6). The null mutants and the overexpressing strains did not present phenotypic significant differences on growth rate, conidiation, or colony appearance compared with the WT (data not shown).

Trichoderma strains were grown on Murashige-Skoog minimal medium and the supernatants were analyzed using a gas chromatography mass spectrometry (GC-MS) system. The obtained chromatograms from each strain were compared to identify differences among the secreted secondary metabolites. These assays allowed us to determine if both OETvCyt 2 strains have differential metabolites at higher concentrations in comparison with the parental and null mutant strains (Fig. 3A). According to the mass spectra obtained from the chromatograms of the OETvCyt 2 strains (Supplementary Fig. S7), peaks a, b, and c correspond to the compounds viridiflorol, tau-muurolol, and $\alpha$-cadino, respectively (Fig. 3B); whereas peaks d and e were unknown compounds of the sesquiterpene type (Fig. 3C). Compound $\mathrm{f}$ (pyrazine [1,2-a] indole-1,4-diene, 2,3-dihydro-2-methyl-3-methylene) was the most abundant; however, this compound is present in all strains (Fig. 3A). These results suggest that TvCyt2 is involved, either directly or indirectly, in the biosynthesis of different secondary metabolites.

\section{TvCyt2 plays a role}

in the $T$. virens antagonistic interactions.

Previously, we observed a different expression pattern of $T v C y t 2$ when $T$. virens was confronted with two anastomosis groups from $R$. solani, suggesting that the compounds produced through the pathway in which TvCyt 2 is participating could play a role in the antagonistic activity against $R$. solani, with different degrees of impact.

Considering that the overexpressing strains produce more secondary metabolites, we decided to analyze the impact of secondary metabolite production in these strains on the antagonistic activity against $R$. solani. As shown in Figure 4, in antibiosis assays, we observed that both anastomosis groups formed similar-sized colonies in the media in which $T$. virens WT had previously been grown. The same result was observed when we used plates on which OETvCyt2 strains were grown. While the colonies formed by the AG2 strain were bigger in the media in which $T v C y t 2$ null mutant strains had been cultivated, this effect was not observed in the AG5 strain. These results indicate that TvCyt 2 is involved in the production of molecules necessary to control $R$. solani AG2 strain.

TvCyt 2 is involved in the production of metabolites that differentially modulate plant defense responses.

The plant defense pathways that Trichoderma spp. are capable of inducing in plants involve both JA and SA (Martínez et al. 2001; Salas-Marina et al. 2011). To examine the possible role for the metabolites produced by the pathway on which TvCyt 2 participates to modulate plant defense responses, we examined the null mutants, the overexpressing strains, and WT

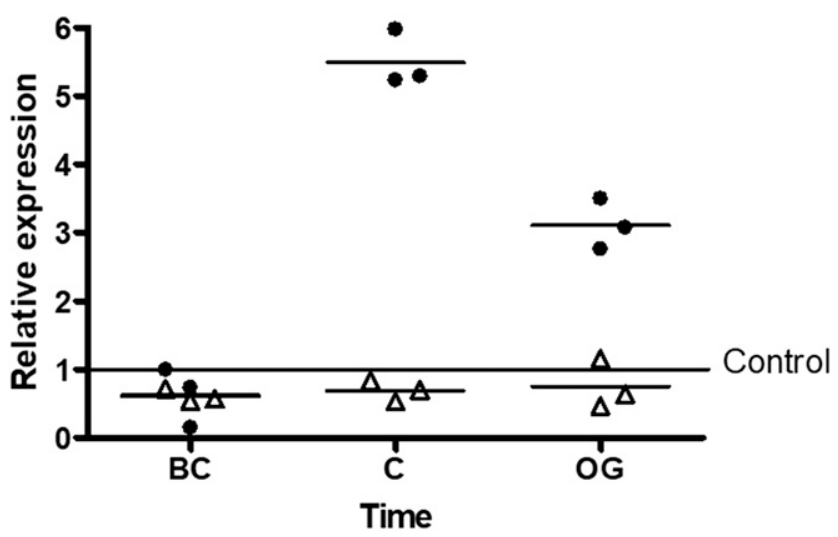

Fig. 2. Expression of $T v C y t 2$ is differentially controlled in response of two antagonistic groups of Rhizoctonia solani. Direct confrontations between Trichoderma and two anastomosis groups from $R$. solani were carried out. The AG2 group is represented with triangles and the AG5 group with circles. Mycelia were collected before contact between the two fungi (BC), during initial contact (C), and at the time when Trichoderma virens overgrew the phytopathogens (OG). The glyceraldehyde-3-phosphate dehydrogenase gene $(g p d)$ was used as endogenous expression control. The quantitative reverse transcription-polymerase chain reaction assay was done in triplicate with three technical replicates each. Data were analyzed using an analysis of variance nonparametric test with $P<0.05$. Only the expression of $T v C y t 2$ in confrontation with AG5 during the initial contact (C) and the overgrown (OG) had significant differences with respect to the control. 
in interaction with two A. thaliana transgenic lines containing the uidA gene reporter, which encodes for the $\beta$-glucuronidase (GUS) enzyme. One marker line contained the uidA gene under the control of the promoter of Lox2, a gene activated by JA (Schommer et al. 2008). In the second transgenic line, the reporter gene was under the control of the promoter of Prla, which is activated by SA (Shah et al. 1997). A. thaliana transgenic seedlings carrying those markers were cocultivated with each of the strains generated in this work. Although the GUS staining shown by seedlings of the pPrla:uidA and pLox2:uidA lines without interacting with $T$. virens was low (Fig. 5A), the signal was slightly activated in shoots of plants cocultivated with the WT strain; the OETvCyt 2 strain activated pPrla:uidA expression slightly more than the WT strain, while the null mutant strain did not activate pPrla:uidA expression (Fig. 5F to 5H). The JAactivated marker $p$ Lox2: uidA was expressed at a slightly lower level in plants cocultivated with the null mutant strain, as compared with those cocultivated with the WT strain. Interestingly, the OETvCyt2 strain activated $p L o x 2:$ uidA expression at a higher level than the WT strain (Fig. 5B to D). These data indicate that the products generated by $\mathrm{TvCyt} 2$ are involved in triggering A. thaliana responses mainly mediated by JA.

\section{TvCyt 2 has a role in plant growth enhancement mediated by $T$. virens.}

Trichoderma spp. have the ability to promote plant growth through different compounds, including secondary metabolites (Contreras-Cornejo et al. 2009; Salas-Marina et al. 2011; Vinale et al. 2016). To evaluate if TvCyt2 played a relevant role during plant growth promotion, we analyzed Arabidopsis seedlings that had been cocultivated with the overexpressing strains, compared with those cultivated in the presence of the WT strain of $T$. virens. We observed that the OETvCyt 2 strain stimulated lateral root and root-hair formation and increased shoot biomass, indicating a better effect on plant growth than the parental strain at 7 days postinoculation (Supplementary Fig. S8). To corroborate
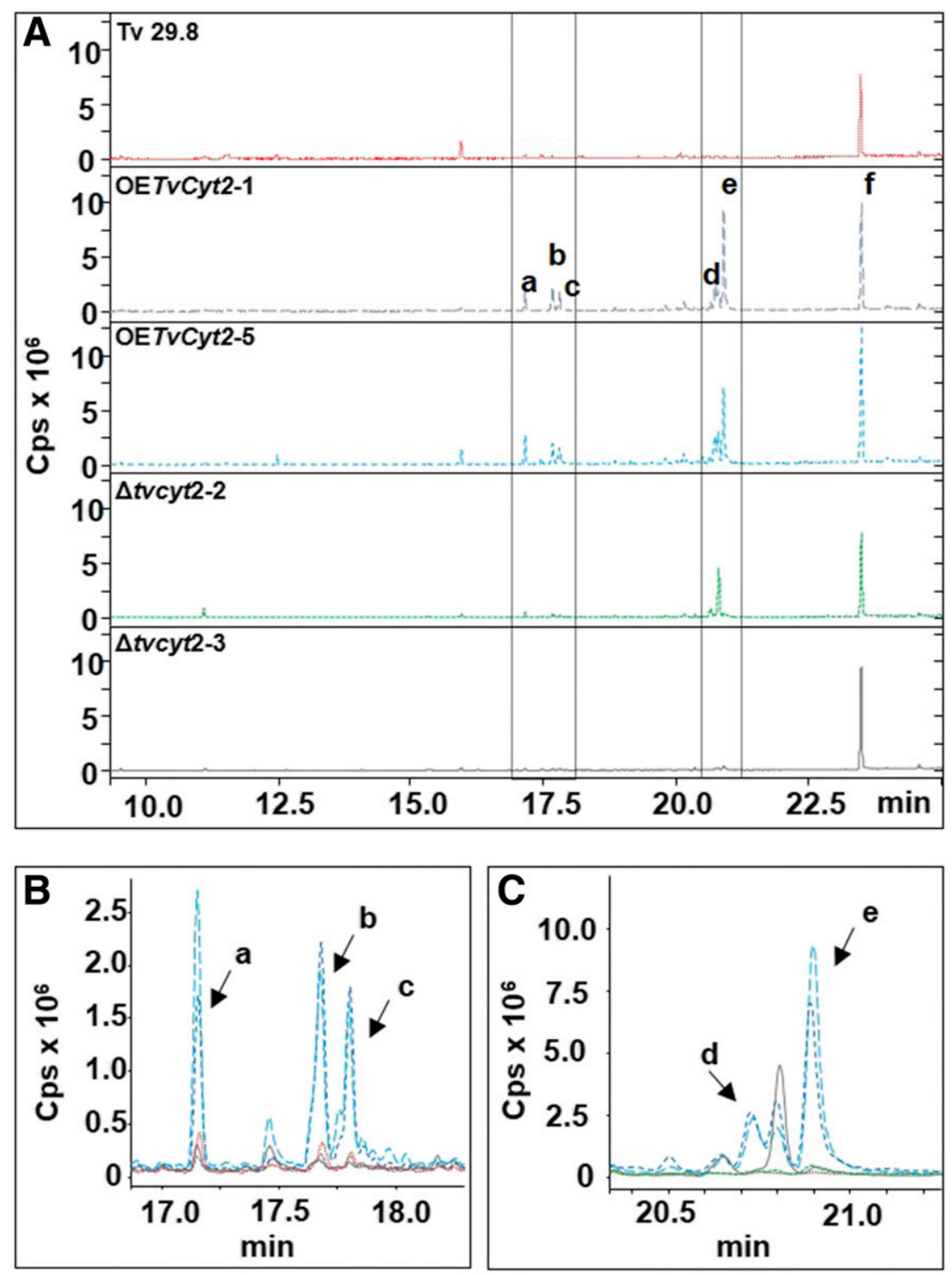

Fig. 3. The alteration in the levels of the product of the $T v C y t 2$ gene modifies the production of secondary metabolites in Trichoderma virens. A, Representative gas chromatography mass spectrometry chromatogram showing different metabolites profile. B, Peaks a, b, and c correspond to the compounds viridiflorol, tau-muurolol, and $\alpha$-cadinol, respectively; $\mathbf{C}$, peaks $\mathrm{d}$ and e were unknown compounds of the sesquiterpene type. Compound $\mathrm{f}$ was pyrazine [1,2-a] indole-1, 4-diene, 2,3-dihydro-2-methyl-3-methylene. The experiment was carried out twice, obtaining similar results. Secondary metabolite profiles obtained from the wild-type strain, two independent null mutants $(\Delta t v c y t 2)$ and two independent overexpressing strains (OETvCyt2) are shown. 
that the metabolites produced by the OETvCyt 2 strains influence plant growth differences, $A$. thaliana seedlings were transferred to plates on which $T$. virens strains had been previously grown for $36 \mathrm{~h}$ and the fungi were then removed. We found that the seedlings exposed to metabolites released by OETvCyt 2 strains showed higher density and length of root hairs compared with seedlings exposed to metabolites produced by the mutant and WT strains (Supplementary Fig. S9). To validate these results and analyze if the effect of $T v C y t 2$ on plant growth promotion can occur in other plant systems, tomato seedlings were cocultivated with the strains described above. As we observed with Arabidopsis, tomato plants in interaction with the OETvCyt 2 strains showed an increase in plant growth, as determined by the size of roots and leaves, and total fresh weight after 3 and 6 weeks postinoculation (Fig. 6). The OETvCyt 2 strains enhanced the number, length, and area of leaves from 3 weeks of interaction (Fig. 6A and D) and the roots were 75\% longer than the roots treated with the WT and null mutant strains as well as the control plants (Fig. 6B). Moreover, the fresh weight was almost double in plants treated with the OETvCyt2 strains, as compared with the
Control T. virens 29.8
OETVCyt2

OE-1
$\Delta$ tvcyt2

$\Delta-2$
$\Delta-3$

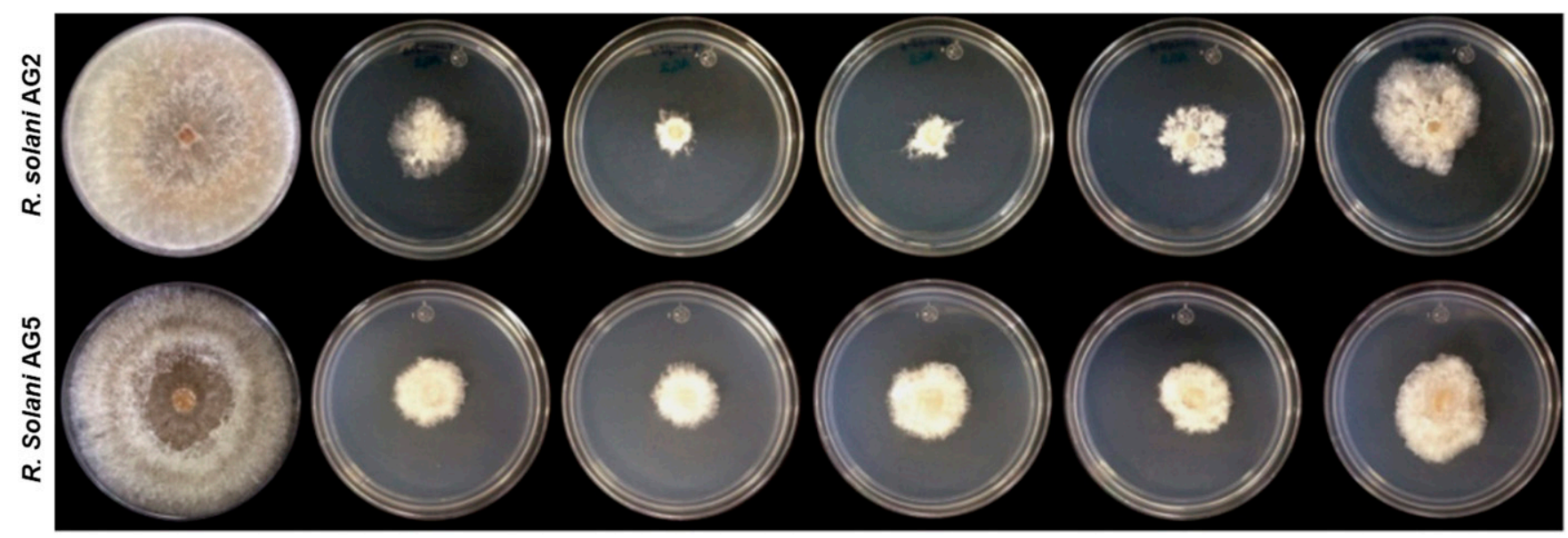

Fig. 4. The overexpression of the $T v C y t 2$ gene improves the antagonistic capacity of Trichoderma virens against Rhizoctonia solani AG2. Antibiosis assays with Trichoderma strains (wild-type, null mutants, or overexpressing strains) and AG2 and AG5 anastomosis groups of the phytopathogen $R$. solani were carried out. Photographs were taken after $72 \mathrm{~h}$ and are representative of three independent experiments.
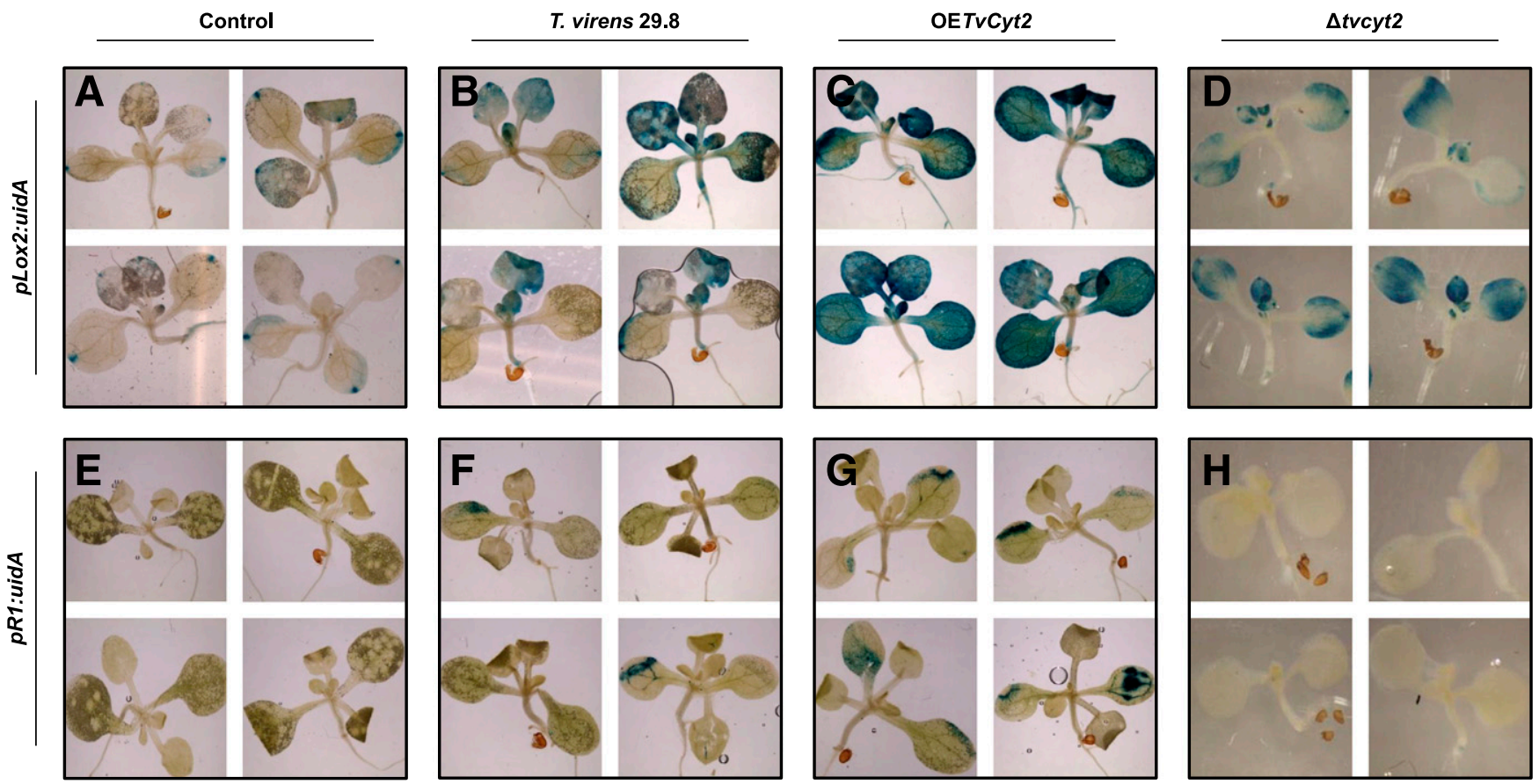

Fig. 5. The metabolites differentially produced by the $T \nu C y t 2$ overexpressing strain activate the jasmonate (JA)-mediated pathway with higher intensity in Arabidopsis thaliana. Transgenic Arabidopsis seedlings containing the $\beta$-glucuronidase (GUS) reporter gene responsive to JA (pLox2:uidA) and salicylate (pPRla:uidA) were $\mathbf{A}$ and $\mathbf{E}$, axenically grown as negative controls or cocultivated with $\mathbf{B}$ and $\mathbf{F}$, Trichoderma virens wild type, $\mathbf{C}$ and $\mathbf{G}$, overexpressing strains, or $\mathbf{D}$ and $\mathbf{H}$, null mutants. GUS activity in seedlings was determined at 7 days of plant-fungi interaction. Photographs show representative individuals of at least 20 stained seedlings. 
other treatments (Fig. 6C). Our results indicate that $T v C y t 2$ is involved in the synthesis of secondary metabolites and these have an important role in both growth and promoting plant defense capabilities, tested in plants commonly used in the laboratory as well as in species of agricultural importance.

\section{DISCUSSION}

The rhizosphere is a complex and dynamic environment shared by a great diversity of organisms that have developed mechanisms to interact with others to ensure their survival. Among other factors, the nature of these interactions will depend on the combination, quality, and quantity of molecular signals that each participant perceives and produces (Bais et al. 2006; Zeilinger et al. 2016). Secondary metabolites play a major role in chemical communication between organisms. In the case of the beneficial associations that Trichoderma spp. establish with plants, a diverse number of molecules are produced (Brotman et al. 2008; Gomes et al. 2015; Mukherjee et al. 2013). It is important to note that, although Trichoderma does not cause plant disease, it shares ortholog genes with pathogenic fungi that could have evolved under selection to allow an optimal Trichoderma-plant interaction and elicit plant defense response without triggering it completely (Horwitz et al. 2013). How the Trichoderma-plant association is controlled on the edge between a beneficial interaction and a pathogenic one is a primary interest in the area.

In this work, we analyzed the participation of $\mathrm{p} 450$ monooxygenase enzyme TvCyt 2 during the interaction of $T$. virens with plants or phytopathogens. We confirmed its role in the synthesis of secondary metabolites, finding that the most abundant compound was pyrazine [1,2-a] indole-1,4-diene, 2,3-dihydro-2methyl-3-methylene; however, this was found in all tested strains, suggesting that TvCyt 2 activity is not related to its synthesis. We found five terpene-like molecules in the overexpressing strains that were absent or present in very low amounts in the WT and null mutant strains. Several studies have demonstrated the properties of sesquiterpenes; their oxygenated forms have a wide spectrum of biological activities, participating as inducers of plant defense mechanisms as well as important antimicrobial properties. This activity has been related with the presence of one or both an unsaturated double bond or oxygen-containing functional group (Kramer and Abraham 2012; Petrelli et al. 2016; Wu et al. 2005). In plant-microbe associations, sesquiterpenes are crucial for the establishment of the interaction, an example is when Laccaria bicolor interacts with Populus or Arabidopsis plants (Ditengou et al. 2015), highlighting the relevance of secondary metabolites in biological interactions.

To determine if those secondary metabolites participate in the associations between Trichoderma spp. and phytopathogens, we analyzed the expression of $T v C y t 2$ in a confrontation with two anastomosis groups of $R$. solani. There were no differences when analyzing anastomosis group AG2, while the gene was up-regulated in the interaction with AG5 until both fungi established contact. Carpenter and collaborators (2008) reported a monooxygenase gene in $T$. hamatum and its homo$\operatorname{logs}$ in $T$. atroviride and $T$. virens that were expressed specifically in response to the plant pathogens Sclerotinia sclerotiorum, $S$. minor, and $S$. cepivorum but not in response to Botrytis cinerea and the expression of the gene did not occur until contact had been established between the two fungal species. These results

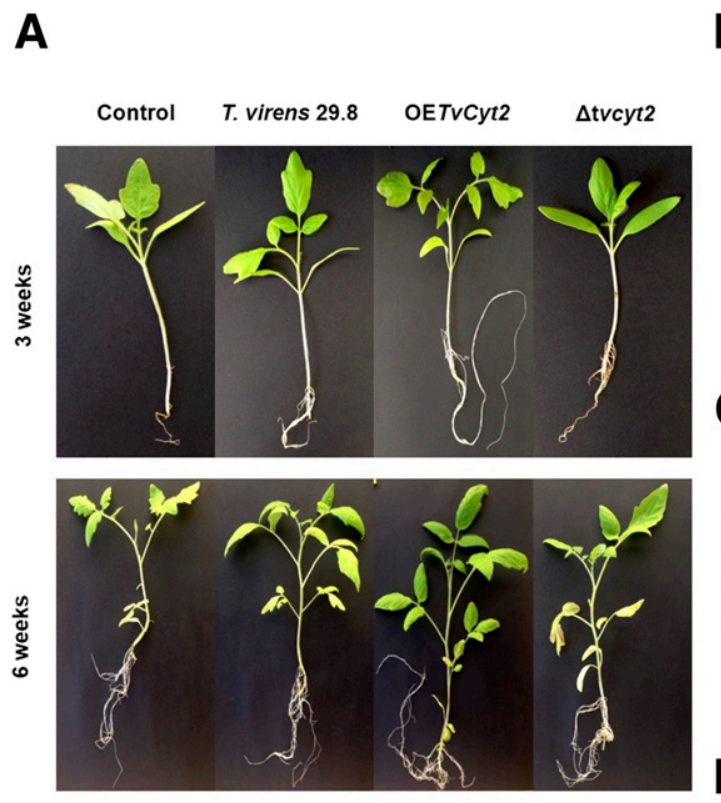

B
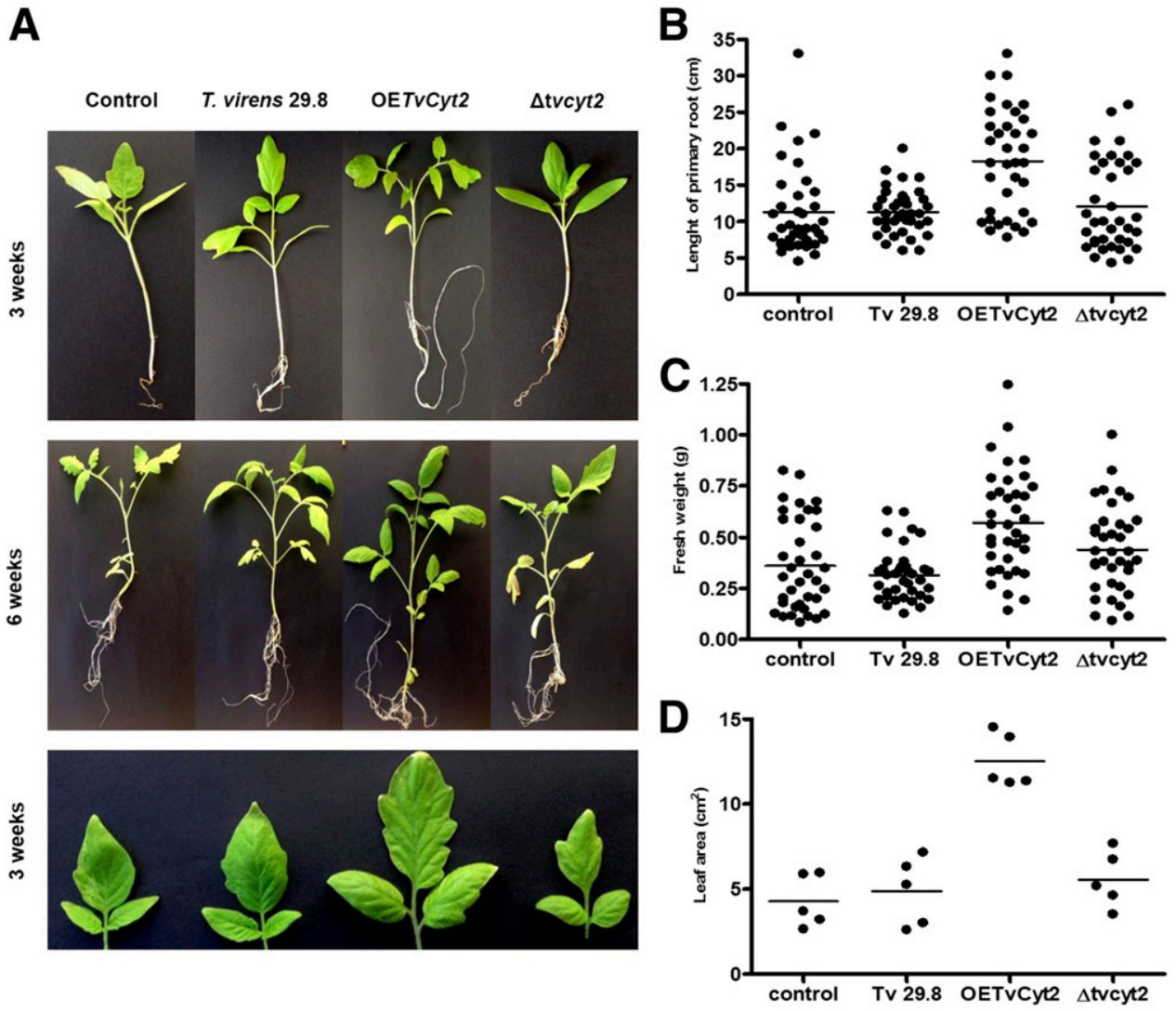

Fig. 6. The $T v C y t 2$ overexpressing strain promotes greater growth in tomato plants. Tomato plants were cocultivated in pots with Trichoderma virens (wild-type strain, null mutants, and overexpressing strains); plants without fungi were used as control. A, Plant size was observed at 3 and 6 weeks posttreatment with the different Trichoderma strains. B, Length of primary root, $\mathbf{C}$, fresh weight, and $\mathbf{D}$, length of leaves were also registered. Data were analyzed using an analysis of variance nonparametric test with $P<0.05$. In all cases, only the treatments with the overexpressing $(\mathrm{OET} V C y t 2)$ strain had significant differences with respect to the control. 
show that this class of genes seems to be regulated in response to specific molecules produced by the host during confrontations with phytopathogens. Nevertheless, the nature or identity of those molecules remains unknown. Additionally, we evaluated whether the loss of function and the overexpression of the TvCyt2 gene had a physiological effect against $R$. solani AG2 and AG5 strains. Antibiosis assays showed that the null mutants were less effective to restrict the growth of the AG2 strain. The expression of $T v C y t 2$ was not increased by the presence of AG2, suggesting that the basal production of metabolites could be enough to antagonize this strain. On the other hand, although $T$. virens increases the expression of $T v C y t 2$ in confrontation with AG5, this one is not susceptible to the secondary metabolites produced through the pathway involving TvCyt2. More antibiosis analyses are needed to determine the range of fungi sensitivity to these metabolites.

During $T$. virens and $A$. thaliana interactions, we observed that, even before there was physical contact between both participants, the expression of $T v C y t 2$ was down-regulated, subsequently increasing until reaching the basal expression levels observed in the control condition. This behavior in gene expression has been observed in epll and Tatrx2 genes reported by Guzmán-Guzmán et al. (2017). Moreover, a transcriptomic analysis of T. atroviride in interactions with $A$. thaliana showed a larger number of downregulated genes than upregulated genes, suggesting that the presence of the plant provides signals participating in the downregulation of some genes normally expressed in the vegetative state, thus starting a chemical communication that contributes to the establishment of the symbiosis (A. Herrera-Estrella personal communication). Once the association has been established, it is probable that the expression of some of these genes returns to their basal levels to contribute to promotion of the interaction.

In other Trichoderma species, it is reported that the disruption of different p450 genes may either induce or reduce the plant defense response, depending on the pathway in which the gene product participates (Cardoza et al. 2014; Malmierca et al. 2012). In our case, the higher activation of the JA-mediated defense response shown by plants that interacted with the OETvCyt 2 strains could represent an advantage for the plant to respond to challenges. Interestingly, Kottb and collaborators (2015), concluded that $A$. thaliana perceives the Trichoderma metabolites as stress compounds and subsequently initiates multilayered adaptations including activation of signaling cascades to withstand this environmental influence. A similar mechanism might be used by Arabidopsis when it is in the presence of Trichoderma OETvCyt 2 strains.

Secondary metabolites synthetized by TvCyt 2 are important for the biocontrol activity of $T$. virens and they participate in inducing plant defense responses. The presence of both fungi and plants has an impact on the expression of the $T v C y t 2$ gene, showing how these processes can establish a network between the organisms and how secondary metabolites play an important role in Trichoderma-host interactions. However, it is necessary to determine the biological behavior in interactions with three or more participants, aimed at representing what could happen in the field. Given the proposed role for gene product TvCyt2, it is important to highlight that the modification of the expression of only this enzyme leads to a drastic change in the interaction of Trichoderma spp. with plant hosts or fungal antagonists, revealing a fine-tuning orchestrated by different molecular signals that work together, leading to successful interactions.

\section{MATERIALS AND METHODS}

Fungal strains and plant species.

The $T$. virens WT strain Tv29.8 and the phytopathogenic strains of $R$. solani AG2 and $R$. solani AG5 were used in this study and were propagated on potato dextrose agar (PDA) (Difco) at $28^{\circ} \mathrm{C}$ Seeds of $A$. thaliana Col-0, a transgenic line, carrying a JAinducible $p$ Lox2:uidA construct (Schommer et al. 2008) and the SA-inducible pPrla:uidA construct (Shah et al. 1997) were used.

\section{Obtaining TvCyt2 null mutants and overexpressing strains.}

Knockout mutants of $T v C y t 2$ were generated following double-joint protocol (Yu et al. 2004). A linear DNA sequence containing a hygromycin $\mathrm{B}$ expression cassette flanked by two DNA fragments, homologous to the $T v C y t 25^{\prime}$ and $3^{\prime}$ regions was obtained. Primers to amplify the open reading frame from $T v C y t 2$ were $5^{\prime}$-GGCATACACGGCACTACGCAC-3' and 5'GGCATACACGGCACTACGCAC- $3^{\prime}$, respectively. Nest primers were $5^{\prime}$-CGTGGCAACAGTCTCCACTG- $3^{\prime}$ and $5^{\prime}$-GCACAA GTCGACAGCGAGGTC-3'. Chimeric primers were 5'-GCCTG TTCCTCTTGTCGGCTTTGCTTAAATGTGCTGCAACCTCT CTCAGGCA3-' and 5' -ACCGTCTCCACGCCGCCGTCAATC TCCTTGGTCGATGGCGATGAAGTTGAAACACG-3'. To overexpress the $T v C y t 2$ gene, the complete $T v C y t 2$ encoding sequence $(1.6 \mathrm{~kb})$ was amplified from cDNA and was cloned into the BamHI and HindIII sites of the pUE08 plasmid (EsquivelNaranjo and Herrera-Estrella 2007). The primers $T \nu C y t 2-\mathrm{F}\left(5^{\prime}-\mathrm{G}\right.$ GATCCATCATGTTCAATATCCTTGTCGC- $3^{\prime}$ ) and $T v C y t 2-\mathrm{R}$ (5'-AAGCTTCATGTGCTCATTCGTGAGTC-3') were used. Protoplasts of $T$. virens 29.8 were transformed with the purified plasmid. All transformants were subjected to at least three rounds of monosporic culture. The identification and selection of the transformants were performed by PCR using the specific internal primers to $T v C y t 2$ and $h p h$ genes mentioned in the next section. Additionally, two more fragments were amplified, a $5^{\prime}$ region amplified using a direct primer localized outside of the replacement construction and a reverse primer localized inside the $h p h$ gene (TvCyt 2 ctrl F: 5'-CCGTGCTACCCAATGTGATAGC-3' and $h p h C$ R: $5^{\prime}$-CCACTAGCTCCAGCCAAGCC-3'), amplifying a product of 3,799 bp. The $3^{\prime}$ region, amplified using a reverse primer localized outside of the replacement construction and a direct primer inside the $h p h$ gene $\left(h p h C\right.$ F: $5^{\prime}$-CGTCCGAGG GCAAAGGAATAG-3' and TvCyt2 ctrl R: 5'-GGGTCTCGA GAACCTCTAGATGG-3') and amplifies a product of 3,857 bp.

\section{Gene analysis by PCR.}

PCR reactions were carried out with $50 \mathrm{ng}$ of DNA as template and 25 amplification cycles. We used the $T v C y t 2$ primers Forward 5'-AAGCATCTACCCGATGGGTTTCCA-3' and Reverse 5'-CGTTTGCCATTTGCCTCTCAACGA-3'. The glyceraldehyde3-phosphatedehydrogenase gene $(g p d)$, a constitutive gene (Carreras-Villaseñor et al. 2013), was used as a control of expression (Forward 5'-GCTGCCGATGGTGAGCTCAAGGG-3' and Reverse 5' - GAGGTCGAGGACACGGCGGGA-3'). To amplify $h p h$ gene, the primers HygF $\left(5^{\prime}\right.$-GATCGACGTTAAC TGATATTGAAGGAG- $\left.3^{\prime}\right)$ and HygR (5'-CTATTCCTTTGCCC TCGGACGAGTGCTG-3') were used.

\section{A. thaliana-Trichoderma interaction assays.}

Seeds of $A$. thaliana Col-0 were surface-sterilized with ethanol four times and were then placed in $0.2 \times$ Murashige-Skoog agar plates, 10 seeds per plate, and were incubated at $24^{\circ} \mathrm{C}$ in a plant growth chamber (MRClab) with 16-h light and 8-h dark cycles. T. virens strains were inoculated 4 days after seed germination (Contreras-Cornejo et al. 2009). Plates were incubated for 3,5 , and 7 days, corresponding to the stage of interaction analyzed, i.e., before contact, contact, and overgrowth. At each time, mycelia from the culture edge $(1 \mathrm{~cm}$ in width) were collected, were frozen, and were subjected to total RNA extraction and further cDNA synthesis. T. virens was grown alone as control. Three independent experiments were performed. 


\section{Tomato-Trichoderma interactions.}

Tomato seeds were germinated on vermiculite, and after three weeks, the roots were incubated, for $90 \mathrm{~min}$, on a $1 \times 10^{6}$ spore suspension with each one of the Trichoderma strains or with water as control. After that, seedlings were transplanted to plots containing sterile soil and were incubated at room temperature. The plants were irrigated with water for 3 weeks. Tomato plants were carefully removed from containers. Plant length was measured with a ruler and fresh weight was measured on an analytical scale. Plants were returned to their pot and, after 1 week, were irrigated with a spore suspension $(1 \times$ $10^{6}$ conidia per milliliter) and, 3 weeks later, plant length was measured again. The area of the leaves was measured using the software ImageJ 3.0 (NIH Image for the Macintosh).

\section{Direct fungal confrontation assays.}

For mycoparasitic confrontation assays to analyze the expression of $T v C y t 2$, T. virens Gv29-8 was inoculated on PDA plates and was grown at $28^{\circ} \mathrm{C}$ in total darkness, confronted with $R$. solani AG5 and AG2 or with itself as a control. The confrontations were carried out on plates covered with a double cellophane membrane, and mycelia from Trichoderma strains were collected before contact occurred between the two fungi, during contact of both strains, and after Trichoderma had overgrown $R$. solani. Three independent experiments for each confrontation were performed.

\section{Antibiosis assays.}

T. virens Gv29-8, null mutants, and overexpressing strains were inoculated on PDA plates covered with a double cellophane membrane and were grown at $28^{\circ} \mathrm{C}$ for $48 \mathrm{~h}$. After that, the cellophane membrane with the mycelia was removed. On the same medium, $R$. solani AG5 and AG2 were grown at $28^{\circ} \mathrm{C}$ for $72 \mathrm{~h}$. $R$. solani AG5 and AG2 grown on PDA were used as controls. Three independent experiments for each assay were performed.

\section{RNA extraction and cDNA synthesis.}

The collected mycelia from plant interactions and from fungal confrontations were frozen immediately in liquid nitrogen. Mycelia were ground to a fine powder and total RNA was isolated using the TRizol method. cDNA was synthesized with RevertAid H Minus first-strand cDNA synthesis kit (Thermo Scientific), following the recommendations of the manufacturer.

\section{Gene expression analysis by qRT-PCR.}

qRT-PCR reactions were performed with Fast SYBR green master mix (Applied Biosystems), using $150 \mathrm{ng}$ of cDNA as template. The gpd gene was used as a housekeeping gene and three technical replicates were analyzed for each type of interaction. The cycle threshold $(\Delta \Delta \mathrm{Ct})$ method was used to analyze the data obtain from the qRT-PCR assays, using StepOne software (Applied Biosystems) to determine the expression of the $T v C y t 2$ gene. The primers for $T v C y t 2$ and $g p d$ genes were the same as those used in PCR assays.

\section{Primer design.}

Primer sequences were designed using the QuantPrime and Primer Quest online tools and the SnapGene program (GSL Biotech, LLC). Primers for qRT-PCR were designed to produce amplicons around $200 \mathrm{bp}$.

\section{Determination of soluble metabolites from $T$. virens.}

Conidia from $T$. virens strains $\left(1 \times 10^{6}\right)$ were inoculated in $100 \mathrm{ml}$ of $0.2 \times$ Murashige-Skoog and were incubated at $28^{\circ} \mathrm{C}$ for $48 \mathrm{~h}$. The medium was filtered and lyophilized. The samples were recovered, were resuspended in $10 \mathrm{ml}$ of water, and were vortexed. Samples of $5 \mathrm{ml}$ were taken and $2 \mathrm{ml}$ of ethyl acetate was added to each. The organic phase was transferred to new tubes, the liquid was evaporated, and a second extraction was done, using $200 \mu \mathrm{l}$ of ethyl acetate. The extracts were analyzed using a GC-MS system (Bruker 456 GC) equipped with a DB$5 \mathrm{MS}$ column $(30 \mathrm{~m}, 0.2 \mathrm{~mm} \mathrm{ID} \times 0.25 \mu \mathrm{m})$ capillary column and connected to a SCION TQ mass spectrometer (Bruker). The column temperature program started at $40^{\circ} \mathrm{C}$ for $2 \mathrm{~min}$ and was increased to $320^{\circ} \mathrm{C}$ at a rate of $10^{\circ} \mathrm{C}$ per minute, and the final temperature was maintained for $5 \mathrm{~min}$. The transfer line temperature and the ion source were set to $250^{\circ} \mathrm{C}$. The carrier gas (helium, 5.0) was set to $1 \mathrm{ml}$ per minute. Internal electron ionization $(70 \mathrm{eV})$ was performed and mass spectra were recorded in the range 40 to $500 \mathrm{~m} / \mathrm{z}$. Data were processed using Bruker MSWS software and were re-evaluated using AMDIS software. Soluble compounds were identified by comparison of the mass spectra with the NIST 11MS library data.

\section{Statistical analysis.}

A Kruskal-Wallis and a Dunn's post hoc test was carried out to compare the data obtained from the assays, using GraphPad Prism 4 software (GraphPad Software, Inc.). Experiments were done at least in triplicate with three technical replicates.

\section{ACKNOWLEDGMENTS}

The authors thank the Laboratorio Nacional UG-CONACYT (project 123732) and I. Macías for technical support during the development of this work and K. R. Valtierra, who generated the Trichoderma strains containing the tagged version of TvCyt2 with mCherry. We thank A. Herrera-Estrella and B. Franco-Bárcenas for critically reading the manuscript. C. A. Ramírez Valdespino and Ma. D. Porras-Troncoso are indebted to CONACyT (Consejo Nacional de Ciencia y Tecnología) fellowships. This study was supported by CONACyT grant CB-1168612 conferred to V. Olmedo-Monfil and by grants 527/2015, 768/2016, and CIFOREA 2016-17 from the University of Guanajuato.

\section{LITERATURE CITED}

Aro, N., Ilmén, M., Saloheimo, A., and Penttilä, M. 2003. ACEI of Trichoderma reesei is a repressor of cellulase and xylanase expression. Appl. Environ. Microbiol. 69:56-65.

Aro, N., Saloheimo, A., Ilmén, M., and Penttilä, M. 2001. ACEII, a novel transcriptional activator involved in regulation of cellulase and xylanase genes of Trichoderma reesei. J. Biol. Chem. 276:24309-24314.

Atanasova, L., Le Crom, S., Gruber, S., Coulpier, F., Seidl-Seiboth, V., Kubicek, C. P., and Druzhinina, I. S. 2013. Comparative transcriptomics reveals different strategies of Trichoderma mycoparasitism. BMC Genomics $14: 121$.

Bairoch, A. 1991. PROSITE: A dictionary of sites and patterns in proteins. Nucleic Acids Res. 19 (Suppl):2241-2245.

Bais, H. P., Weir, T. L., Perry, L. G., Gilroy, S., and Vivanco, J. M. 2006. The role of root exudates in rhizosphere interactions with plants and other organisms. Annu. Rev. Plant Biol. 57:233-266.

Brito, J. P., Ramada, M. H., de Magalhães, M. T., Silva, L. P., and Ulhoa, C. J. 2014. Peptaibols from Trichoderma asperellum TR356 strain isolated from Brazilian soil. Springerplus 3:600.

Brotman, Y., Briff, E., Viterbo, A., and Chet, I. 2008. Role of swollenin, an expansin-like protein from Trichoderma, in plant root colonization. Plant Physiol. 147:779-789.

Cai, F., Yu, G., Wang, P., Wei, Z., Fu, L., Shen, Q., and Chen, W. 2013. Harzianolide, a novel plant growth regulator and systemic resistance elicitor from Trichoderma harzianum. Plant Physiol. Biochem. 73:106-113.

Cardoza, R. E., Malmierca, M. G., and Gutiérrez, S. 2014. Overexpression of erg1 gene in Trichoderma harzianum CECT 2413: Effect on the induction of tomato defence-related genes. J. Appl. Microbiol. 117:812-823.

Carpenter, M. A., Ridgway, H. J., Stringer, A. M., Hay, A. J., and Stewart, A. 2008. Characterisation of a Trichoderma hamatum monooxygenase gene involved in antagonistic activity against fungal plant pathogens. Curr. Genet. 53:193-205.

Carreras-Villaseñor, N., Esquivel-Naranjo, E. U., Villalobos-Escobedo, J. M., Abreu-Goodger, C., and Herrera-Estrella, A. 2013. The RNAi machinery regulates growth and development in the filamentous fungus Trichoderma atroviride. Mol. Microbiol. 89:96-112. 
Chacón, M. R., Rodríguez-Galán, O., Benítez, T., Sousa, S., Rey, M., Llobell, A., and Delgado-Jarana, J. 2007. Microscopic and transcriptome analyses of early colonization of tomato roots by Trichoderma harzianum. Int. Microbiol. 10:19-27.

Collemare, J., Pianfetti, M., Houlle, A. E., Morin, D., Camborde, L., Gagey, M. J., Barbisan, C., Fudal, I., Lebrun, M. H., and Böhnert, H. U. 2008. Magnaporthe grisea avirulence gene ACE1 belongs to an infectionspecific gene cluster involved in secondary metabolism. New Phytol. 179:196-208.

Conrath, U. 2011. Molecular aspects of defence priming. Trends Plant Sci. 16:524-531.

Contreras-Cornejo, H. A., Macías-Rodríguez, L., Beltrán-Peña, E., HerreraEstrella, A., and López-Bucio, J. 2011. Trichoderma-induced plant immunity likely involves both hormonal- and camalexin-dependent mechanisms in Arabidopsis thaliana and confers resistance against necrotrophic fungi Botrytis cinerea. Plant Signal. Behav. 6:1554-1563.

Contreras-Cornejo, H. A., Macías-Rodríguez, L., Cortés-Penagos, C., and López-Bucio, J. 2009. Trichoderma virens, a plant beneficial fungus, enhances biomass production and promotes lateral root growth through an auxin-dependent mechanism in Arabidopsis. Plant Physiol. 149: 1579-1592.

Cortés, C., Gutiérrez, A., Olmedo, V., Inbar, J., Chet, I., and HerreraEstrella, A. 1998. The expression of genes involved in parasitism by Trichoderma harzianum is triggered by a diffusible factor. Mol. Gen. Genet. 260:218-225.

Črešnar, B., and Petrič, S. 2011. Cytochrome P450 enzymes in the fungal kingdom. Biochim. Biophys. Acta 1814:29-35.

Cubero, B., and Scazzocchio, C. 1994. Two different, adjacent and divergent zinc finger binding sites are necessary for CREA-mediated carbon catabolite repression in the proline gene cluster of Aspergillus nidulans. EMBO J. 13:407-415.

Deepika, V. B., Murali, T. S., and Satyamoorthy, K. 2016. Modulation of genetic clusters for synthesis of bioactive molecules in fungal endophytes: A review. Microbiol. Res. 182:125-140.

Ditengou, F. A., Müller, A., Rosenkranz, M., Felten, J., Lasok, H., van Doorn, M. M., Legué, V., Palme, K., Schnitzler, J. P., and Polle, A. 2015. Volatile signalling by sesquiterpenes from ectomycorrhizal fungi reprogrammes root architecture. Nat. Commun. 6:6279.

Durairaj, P., Hur, J.-S., and Yun, H. 2016. Versatile biocatalysis of fungal cytochrome P450 monooxygenases. Microb. Cell Fact. 15:125.

Esquivel-Naranjo, E. U., and Herrera-Estrella, A. 2007. Enhanced responsiveness and sensitivity to blue light by $b l r-2$ overexpression in Trichoderma atroviride. Microbiology 153:3909-3922.

Gomes, E. V., Costa, M. N., de Paula, R. G., de Azevedo, R. R., da Silva F. L., Noronha, E. F., Ulhoa, C. J., Monteiro, V. N., Cardoza, R. E., Gutiérrez, S., and Silva, R. N. 2015. The cerato-platanin protein Epl-1 from Trichoderma harzianum is involved in mycoparasitism, plant resistance induction and self cell wall protection. Sci. Rep. 5:17998.

Guzmán-Guzmán, P., Alemán-Duarte, M. I., Delaye, L., Herrera-Estrella, A., and Olmedo-Monfil, V. 2017. Identification of effector-like proteins in Trichoderma spp. and role of a hydrophobin in the plant-fungus interaction and mycoparasitism. BMC Genet. 18:16.

Hermosa, R., Viterbo, A., Chet, I., and Monte, E. 2012. Plant-beneficial effects of Trichoderma and of its genes. Microbiology 158:17-25.

Hogenhout, S. A., Van der Hoorn, R. A., Terauchi, R., and Kamoun, S. 2009. Emerging concepts in effector biology of plant-associated organisms. Mol. Plant-Microbe Interact. 22:115-122.

Horwitz, B. A., Kosti, I., Glaser, F., and Mukherjee, M. 2013. Trichoderma genomes: A vast reservoir of potential elicitor proteins. Pages 195-208 in: Trichoderma Biology and Applications. P. K.Mukherjee, ed. C. A. B. International, London.

Kottb, M., Gigolashvili, T., Großkinsky, D. K., and Piechulla, B. 2015. Trichoderma volatiles effecting Arabidopsis: From inhibition to protection against phytopathogenic fungi. Front. Microbiol. 6:995.

Kramer, R., and Abraham, W. R. 2012. Volatile sesquiterpenes from fungi: What are they good for? Phytochem. Rev. 11:15-37.

Larkin, J. C. 1994. Isolation of a cytochrome P450 homologue preferentially expressed in developing inflorescences of Zea mays. Plant Mol. Biol. 25: 343-353.

Lorito, M., Mach, R. L., Sposato, P., Strauss, J., Peterbauer, C. K., and Kubicek, C. P. 1996. Mycoparasitic interaction relieves binding of the Cre1 carbon catabolite repressor protein to promoter sequences of the ech42 (endochitinase-encoding) gene in Trichoderma harzianum. Proc. Natl. Acad. Sci. U.S.A. 93:14868-14872.

Lorito, M., Woo, S. L., Harman, G. E., and Monte, E. 2010. Translational research on Trichoderma: From 'omics to the field. Annu. Rev. Phytopathol. 48:395-417.
Malmierca, M. G., Cardoza, R. E., Alexander, N. J., McCormick, S. P., Hermosa, R., Monte, E., and Gutiérrez, S. 2012. Involvement of Trichoderma trichothecenes in the biocontrol activity and induction of plant defense-related genes. Appl. Environ. Microbiol. 78:4856-4868.

Martínez, C., Blanc, F., Le Claire, E., Besnard, O., Nicole, M., and Baccou, J. C. 2001. Salicylic acid and ethylene pathways are differentially activated in melon cotyledons by active or heat-denatured cellulase from Trichoderma longibrachiatum. Plant Physiol. 127:334-344.

McCormick, S. P., Harris, L. J., Alexander, N. J., Ouellet, T., Saparno, A., Allard, S., and Desjardins, A. E. 2004. Tri1 in Fusarium graminearum encodes a P450 oxygenase. Appl. Environ. Microbiol. 70:2044-2051.

Meek, I. B., Peplow, A. W., Ake, C., Jr., Phillips, T. D., and Beremand, M. N. 2003. Tri 1 encodes the cytochrome P450 monooxygenase for C-8 hydroxylation during trichothecene biosynthesis in Fusarium sporotrichioides and resides upstream of another new Tri gene. Appl. Environ. Microbiol. 69:1607-1613.

Morán-Diez, M. E., Trushina, N., Lamdan, N. L., Rosenfelder, L., Mukherjee, P. K., Kenerley, C. M., and Horwitz, B. A. 2015. Hostspecific transcriptomic pattern of Trichoderma virens during interaction with maize or tomato roots. BMC Genomics 16:8.

Mukherjee, M., Horwitz, B. A., Sherkhane, P. D., Hadar, R., and Mukherjee, P. K. 2006. A secondary metabolite biosynthesis cluster in Trichoderma virens: Evidence from analysis of genes underexpressed in a mutant defective in morphogenesis and antibiotic production. Curr. Genet. 50:193-202.

Mukherjee, P. K., Horwitz, B. A., Herrera-Estrella, A., Schmoll, M., and Kenerley, C. M. 2013. Trichoderma research in the genome era. Annu. Rev. Phytopathol. 51:105-129.

Nebert, D. W., and González, F. J. 1987. P450 genes: Structure, evolution, and regulation. Annu. Rev. Biochem. 56:945-993.

O’Connell, R. J., Thon, M. R., Hacquard, S., Amyotte, S. G., Kleemann, J., Torres, M. F., Damm, U., Buiate, E. A., Epstein, L., Alkan, N., Altmüller, J., Alvarado-Balderrama, L., Bauser, C. A., Becker, C., Birren, B. W., Chen, Z., Choi, J., Crouch, J. A., Duvick, J. P., Farman, M. A., Gan, P., Heiman, D., Henrissat, B., Howard, R. J., Kabbage, M., Koch, C. Kracher, B., Kubo, Y., Law, A. D., Lebrun, M. H., Lee, Y. H., Miyara, I., Moore, N., Neumann, U., Nordström, K., Panaccione, D. G., Panstruga, R., Place, M., Proctor, R. H., Prusky, D., Rech, G., Reinhardt, R., Rollins, J. A., Rounsley, S., Schardl, C. L., Schwartz, D. C., Shenoy, N., Shirasu, K., Sikhakolli, U. R., Stüber, K., Sukno, S. A., Sweigard, J. A., Takano, Y., Takahara, H., Trail, F., van der Does, H. C., Voll, L. M., Will, I., Young, S., Zeng, Q., Zhang, J., Zhou, S., Dickman, M. B., SchulzeLefert, P., Ver Loren van Themaat, E., Ma, L. J., and Vaillancourt, L. J. 2012. Lifestyle transitions in plant pathogenic Colletotrichum fungi deciphered by genome and transcriptome analyses. Nat. Genet. 44: 1060-1065

Ortíz-Castro, R., Contreras-Cornejo, H. A., Macías-Rodríguez, L., and López-Bucio, J. 2009. The role of microbial signals in plant growth and development. Plant Signal. Behav. 4:701-712.

Park, J. W., Reed, J. R., and Backes, W. L. 2015. The localization of cytochrome P450s CYP1A1 and CYP1A22 into different lipid microdomains is governed by their $\mathrm{N}$-terminal and internal protein regions. J. Biol. Chem. 290:29449-29460.

Park, J. W., Reed, J. R., Brignac-Huber, L. M., and Backes, W. L. 2014. Cytochrome $\mathrm{P} 450$ system proteins reside in different regions of the endoplasmic reticulum. Biochem. J. 464:241-249.

Petrelli, R., Orsomando, G., Sorci, L., Maggi, F., Ranjbarian, F., Biapa Nya, P. C., Petrelli, D., Vitali, L. A., Lupidi, G., Quassinti, L., Bramucci, M., Hofer, A., and Cappellacci, L. 2016. Biological activities of the essential oil from Erigeron floribundus. Molecules 21:1065.

Rubio, M. B., Domínguez, S., Monte, E., and Hermosa, R. 2012. Comparative study of Trichoderma gene expression in interactions with tomato plants using high-density oligonucleotide microarrays. Microbiology 158:119-128.

Salas-Marina, M. A., Silva-Flores, M. A., Uresti-Rivera, E. E., CastroLongoria, E., Herrera-Estrella, A., and Casas-Flores, S. 2011. Colonization of Arabidopsis roots by Trichoderma atroviride promotes growth and enhances systemic disease resistance through jasmonic acid/ethylene and salicylic acid pathways. Eur. J. Plant Pathol. 131: 15-26.

Schommer, C., Palatnik, J. F., Aggarwal, P., Chételat, A., Cubas, P., Farmer, E. E., Nath, U., and Weigel, D. 2008. Control of jasmonate biosynthesis and senescence by miR319 targets. PLoS Biol. 6:e230.

Shah, J., Tsui, F., and Klessig, D. F. 1997. Characterization of a salicylic acid-insensitive mutant (sai1) of Arabidopsis thaliana, identified in a selective screen utilizing the SA-inducible expression of the $t m s 2$ gene. Mol. Plant-Microbe Interact. 10:69-78. 
Sharma, S., Kumari, I., Hussain, R., Ahmed, M., and Akhter, Y. 2017. Species specific substrates and products choices of 4- $O$-acetyltransferase from Trichoderma brevicompactum. Enzyme Microb. Technol. 104:29-36.

Shoresh, M., Harman, G. E., and Mastouri, F. 2010. Induced systemic resistance and plant responses to fungal biocontrol agents. Annu. Rev. Phytopathol. 48:21-43.

Tijerino, A., Cardoza, R. E., Moraga, J., Malmierca, M. G., Vicente, F., Aleu, J., Collado, I. G., Gutiérrez, S., Monte, E., and Hermosa, R. 2011. Overexpression of the trichodiene synthase gene tri5 increases trichodermin production and antimicrobial activity in Trichoderma brevicompactum. Fungal Genet. Biol. 48:285-296.

Verhagen, B., Trotel-Aziz, P., Jeandet, P., Baillieul, F., and Aziz, A. 2011. Improved resistance against Botrytis cinerea by grapevine-associated bacteria that induce a prime oxidative burst and phytoalexin production. Phytopathology 101:768-777.

Vinale, F., Flematti, G., Sivasithamparam, K., Lorito, M., Marra, R., Skelton, B. W., and Ghisalberti, E. L. 2009. Harzianic acid, an antifungal and plant growth promoting metabolite from Trichoderma harzianum. J. Nat. Prod. 72:2032-2035.

Vinale, F., Strakowska, J., Mazzei, P., Piccolo, A., Marra, R., Lombardi, N., Manganiello, G., Pascale, A., Woo, S. L., and Lorito, M. 2016. Cremenolide, a new antifungal, 10-member lactone from Trichoderma cremeum with plant growth promotion activity. Nat. Prod. Res. 6419: 1-7.

Vos, C. M., De Cremer, K., Cammue, B. P., and De Coninck, B. 2015. The toolbox of Trichoderma spp. in the biocontrol of Botrytis cinerea disease. Mol. Plant Pathol. 16:400-412.

Werck-Reichhart, D., and Feyereisen, R. 2000. Cytochromes P450: A success story. Genome Biol. 1:REVIEWS3003.
Woo, S. L., Scala, F., Ruocco, M., and Lorito, M. 2006. The molecular biology of the interactions between Trichoderma spp., phytopathogenic fungi, and plants. Phytopathology 96:181-185.

Wu, C. L., Chien, S. C., Wang, S. Y., Kuo, Y. H., and Chang, S. T. 2005. Structure-activity relationships of cadinane-type sesquiterpene derivatives against wood-decay fungi. Holzforschung 59:620-627.

Yi, M., and Valent, B. 2013. Communication between filamentous pathogens and plants at the biotrophic interface. Annu. Rev. Phytopathol. 51:587-611.

Yu, J.-H., Hamari, Z., Han, K.-H., Seo, J.-A., Reyes-Domínguez, Y., and Scazzocchio, C. 2004. Double-joint PCR: A PCR-based molecular tool for gene manipulations in filamentous fungi. Fungal Genet. Biol. 41:973-981.

Zeilinger, S., Galhaup, C., Payer, K., Woo, S. L., Mach, R. L., Fekete, C., Lorito, M., and Kubicek, C. P. 1999. Chitinase gene expression during mycoparasitic interaction of Trichoderma harzianum with its host. Fungal Genet. Biol. 26:131-140.

Zeilinger, S., Gupta, V. K., Dahms, T. E., Silva, R. N., Singh, H. B., Upadhyay, R. S., Gomes, E. V., Tsui, C. K., and Nayak S, C. 2016. Friends or foes? Emerging insights from fungal interactions with plants. FEMS Microbiol. Rev. 40:182-207.

Zhai, X., Jia, M., Chen, L., Zheng, C. J., Rahman, K., Han, T., and Qin, L. P 2017. The regulatory mechanism of fungal elicitor-induced secondary metabolite biosynthesis in medical plants. Crit. Rev. Microbiol. 43:238-261.

\section{AUTHOR-RECOMMENDED INTERNET RESOURCES}

Primer Quest tool: http://www.idtdna.com/Primerquest/Home/Index QuantPrime website: http://quantprime.mpimp-golm.mpg.de 